\title{
Treatment of Cyclic Vomiting Syndrome: Is Bathing a Panacea or Is It in Hot Water?
}

\author{
Vikram Rangan $^{1} \cdot$ Andrew Ukleja $^{1}$
}

Published online: 10 July 2020

(c) Springer Science+Business Media, LLC, part of Springer Nature 2020

Cyclic vomiting syndrome (CVS) and cannabinoid hyperemesis syndrome (CHS) are two related disorders with incompletely understood pathogenesis. Both syndromes share similar clinical manifestations, though CHS according to the Rome IV criteria requires prolonged excessive cannabis use [1]. CHS may be misdiagnosed for a prolonged period if cannabis use is not accurately reported, which is common among patients using or abusing cannabis. Recent studies have shown a significant increase in cannabis use, especially among teenagers [2], accompanied by increases of both ED visits for cyclic vomiting [3] as well as hospitalizations for vomiting associated with cannabis use disorders [4]. The increasing prevalence and challenges in managing these conditions provide a clear impetus for studies aimed at better understanding their pathophysiology and potential therapies.

Patients often use hot water bathing for temporary relief of prodromal symptoms as well as subsequent nausea and vomiting. In this issue of Digestive Diseases and Sciences, Venkatesan et al. utilized questionnaire data in order to report detailed clinical characteristics of hot water bathing patterns in 111 patients carrying a clinical diagnosis of cyclic vomiting syndrome [5]. Seventy-three percent utilized hot water bathing for alleviating the prodromal and GI symptoms (nausea, vomiting, abdominal pain and diarrhea). Most of these individuals reported a significant improvement in symptoms of both the prodromal and emetic phases of a flare, including not only nausea, vomiting, and abdominal pain, but also symptoms associated with panic. The authors stated that none of their patients met criteria for cannabinoid hyperemesis syndrome, though of note, $17 \%$ of those utilizing hot water bathing were characterized by the authors as

Vikram Rangan

vrangan@bidmc.harvard.edu

1 Division of Gastroenterology, Department of Medicine, Beth Israel Deaconess Medical Center and Harvard Medical School, 330 Brookline Avenue, Dana 535, Boston, MA 02215, USA regular cannabis users, with reported usage 4 or more times per week. The authors hypothesize that the improvement in symptoms may be mediated by increased release of endocannabinoids acting on the CB1 subtype of cannabinoid receptors. The authors also posit a role for the vanilloid receptor TRPV1, which is also targeted by endocannabinoids, and may also be activated directly by heat.

A key question raised by this study is whether the presence of hot water bathing for amelioration of nausea and vomiting is sufficiently correlated with CVS in order to obviate the need for certain diagnostic tests in presumptive CVS patients, who often undergo extensive evaluation prior to arriving at a formal diagnosis. Indeed, the authors note that hot water bathing is not a common practice in any other known disease. An upper endoscopy and evaluation for metabolic causes are recommended for all suspected CVS cases [6]. Current guidelines do not clearly recommend a gastric emptying study or cross-sectional imaging [6], although these studies are often performed in order to exclude gastroparesis and structural abnormalities in those with suspected CVS. Given the clear association between hot water bathing and CVS noted in this study, the question arises as to whether the presence of hot water bathing may provide a sufficiently robust clinical clue that symptoms are more likely to fall on the CVS spectrum, and hence reduce the ordering of studies that are often expensive, cumbersome, and associated with radiation exposure.

This article also raises questions about the distinction between CVS and CHS, which has been debated in the existing literature. While earlier case series on CHS proposed that hot water bathing was a behavior unique to CHS, a more recent review article [7] suggested that it was a behavior common to both conditions. In another article, Venkatesan et al. [8] proposed that CHS may in fact represent a subset of CVS rather than a distinct entity.

This study further suggests that hot water bathing cannot be reliably used to distinguish CVS from CHS. Nevertheless, it is quite possible that many patients within the 
regular cannabis use (CU) group of this study, who were using cannabis 4 times per week or more, may indeed have met criteria for CHS. The majority of patients $(64 \%)$ in the CU group were males versus only $24 \%$ males in the occasional or non-users (OU) group, in line with our suspicion that clinical manifestations in the $\mathrm{CU}$ group were most likely related to cannabis abuse, acknowledging that male gender is a known risk factor for developing CU disorders [9]. Underreporting of cannabis use could be an issue as well, as the low response rate may have reflected not only an inherent limitation of an emailed survey, but also concern on the part of some participants about privacy and the reporting of details of their cannabis use (the authors note this limitation as well). Even among respondents, there may thus have been some underreporting of cannabis use. Ultimately, the only truly reliable differentiating factor between CHS and CVS is the patient's response to a prolonged trial of marijuana cessation, which was not assessed by the questionnaire utilized in this study, since the distinction between CHS and CVS in this study was made in the clinic.

Regardless of the distinction between CVS and CHS, by showing that hot water bathing can be therapeutic for vomiting symptoms in patients with varying levels of cannabis use, the authors suggest the pathogenesis of vomiting may be similar at various different points along the cannabis use spectrum, with the $\mathrm{eCB} / \mathrm{CB} 1 \mathrm{R}$ signaling pathway playing a role in vomiting, and importantly contributing to the therapeutic effect of hot water bathing. Thus, a key question is whether similar therapeutics may be applied to both CVS and CHS. Venkatesan et al. [8] previously suggested that tricyclic antidepressants (TCA), often used on a prophylactic basis for CVS, may be used for CHS as well. Yet, others have noted a lack of evidence for the use of CVS therapeutic strategies in the management of CHS [7], and recognized that chronic marijuana use is a risk factor for non-response to TCAs [10].

Similarly, the question arises as to whether a trial of cannabis cessation should be the first-line of treatment even in occasional marijuana users who meet criteria for CVS but not CHS (which stipulates "prolonged, excessive cannabis use"). The most recent recommendations on the management of CVS do not mention a trial of complete cannabis cessation in occasional marijuana users [6]. It is unclear whether the findings of this study can be used to support prolonged marijuana cessation as a first-line therapeutic trial in CVS patients with occasional use. While this study did find that hot water bathing provided clinical relief in both occasional and regular cannabis users, it also noted clinical differences between the two groups. In addition to the previously noted increased proportion of males in the CU group, regular cannabis users also reported more panic type symptoms and more symptoms during the prodromal phase, had a slower response to hot water exposure, a shorter time to symptom relapse, and were more likely to utilize "very hot" water to relieve symptoms $(50 \%$ vs. $16 \%$ ), perhaps suggesting more pronounced effects of chronic marijuana use on CVS symptoms. Whether the differences in clinical presentations and in hot water bathing patterns between occasional and chronic marijuana users indicate the presence of two distinct disease phenotypes, and whether a trial of marijuana cessation is likely to have the same utility in both groups, is an important question for a future study. Further information on those patients in the study who did not utilize hot water bathing-particularly knowing whether most of these individuals were non-cannabis users-would provide further insight into the link between cannabis use and vomiting, as would separate sub-analyses of patients who were not marijuana users at all.

Additional important clinical details that may provide insight on hot water bathing behavior include how patients learned about hot water bathing, and whether this is indeed a self-learned behavior in most cases. We can only speculate that most cannabis users are clearly aware of hot water bathing therapy for CHS and treat themselves, as conventional anti-emetics are frequently ineffective. Another interesting question is how often health care providers recommend hot water bathing to their patients with both CVS and CHS. Indeed, the results of this study may even support the use of hot water bathing in clinical practice, though of note, $6 \%$ of patients in the study who used hot water bathing sustained superficial burns, raising a major concern about safety of this behavior as well as physical alertness of individuals during bathing. Knowledge of the timing between cannabis use and the onset of CVS symptoms as well as the timing of hot water bathing in relation to cannabis use would provide further insight into the role of both cannabis use and hot water bathing in the exacerbation and alleviation of vomiting.

This important study has shown that hot water bathing temporary relieves multiple symptoms during both the prodromal and emetic phases of CVS in a population of individuals with varying levels of marijuana use. In so doing, it provides further insight into the pathogenesis of CVS, raising important questions about whether similar treatments can be utilized throughout the CVS/CHS spectrum. This study will likely lead to further streamlining of treatment recommendations for both CVS and CHS.

\section{Compliance with Ethical Standards}

Conflict of interest Authors declare that they have no conflict of interest. 


\section{References}

1. Stanghellini V, Chan FKL, Hasler WL, et al. Gastroduodenal disorders. Gastroenterology. 2016;150:1380-1392.

2. Yu B, Chen X, Chen X, Yan H. Marijuana legalization and historical trends in marijuana use among US residents aged 12-25: results from the 1979-2016 National Survey on drug use and health. BMC Public Health. 2020;20:156.

3. Kim HS, Monte AA. Colorado Cannabis legalization and its effect on emergency care. Ann Emerg Med. 2016;68:71-75.

4. Patel RS, Patel J, Jaladi PR, Bhimanadham NN, Imran S, Tankersley WE. Burden of persistent vomiting with cannabis use disorder: report from 55,549 hospitalizations in the United States. Psychosomatics. 2019;60:549-555.

5. Rosen S, Diaz R, Garacci Z, Kumar VCS, Thyarala SR, Hillard $\mathrm{CJ}$, et al. Hot-water bathing improves symptoms in patients with cyclic vomiting syndrome and is modulated by chronic cannabis use. Dig Dis Sci. (Epub ahead of print). http://doi.org/10.1007/ s10620-020-06343-x.

6. Venkatesan T, Levinthal DJ, Tarbell SE, et al. Guidelines on management of cyclic vomiting syndrome in adults by the American Neurogastroenterology and Motility Society and the Cyclic
Vomiting Syndrome Association. Neurogastroenterol Motil. 2019;31:e13604.

7. Blumentrath CG, Dohrmann B, Ewald N. Cannabinoid hyperemesis and the cyclic vomiting syndrome in adults: recognition, diagnosis, acute and long-term treatment. Ger Med Sci. 2017;15:Doc06.

8. Venkatesan T, Levinthal DJ, Li BUK, et al. Role of chronic cannabis use: cyclic vomiting syndrome vs cannabinoid hyperemesis syndrome. Neurogastroenterol Motil. 2019;31:e13606.

9. Stinson FS, Ruan WJ, Pickering R, Grant BF. Cannabis use disorders in the USA: prevalence, correlates and co-morbidity. Psychol Med. 2006;36:1447-1460.

10. Hejazi RA, Lavenbarg TH, Foran P, McCallum RW. Who are the nonresponders to standard treatment with tricyclic antidepressant agents for cyclic vomiting syndrome in adults? Aliment Pharmacol Ther. 2010;31:295-301.

Publisher's Note Springer Nature remains neutral with regard to jurisdictional claims in published maps and institutional affiliations. 